\title{
Assessment of Myogenic Power Expenditure Due to Arterial Wall Smooth Muscle Contraction Based upon the Fractal Nature of Vascular Trees
}

\author{
Akira Kamiya*, Masahiro Shibata, Kimiko Yamamoto \\ Research Laboratory for Interdisciplinary Sciences, Tokyo, Japan \\ Email: ${ }^{*}$ akamiya@kamakuranet.ne.jp
}

Received 27 March 2014; revised 30 April 2014; accepted 7 May 2014

Copyright (C) 2014 by authors and Scientific Research Publishing Inc.

This work is licensed under the Creative Commons Attribution International License (CC BY).

http://creativecommons.org/licenses/by/4.0/

(c) (i) Open Access

\begin{abstract}
The purpose of this study is 1) to present a biomechanical model for evaluating the myogenic power expended in an arterial segment due to vascular smooth muscle contraction (VSMC) and 2) to assess the total power expenditure in the entire systemic arterial tree by utilizing the fractal nature of the branching architecture. The model is based on the mechanical equilibrium between the stretch stress exerted by blood pressure inside the vessel lumen and constricting stress elicited by VSMC in the vascular wall. An expression for myogenic power expenditure is formulated for a unit wall mass as a function of the internal vessel radius and extent of strain. This expression was then integrated over selected range of vessel radii, by taking into account of the fractal nature of the branching structure. When the total myogenic power expended in the systemic arterial tree in rat at the moderate strain level is converted to the oxygen consumption rate, it amounts to approximately $18 \%$ of the whole body oxygen consumption rate. This suggests that the mechanical power expenditure due to VSMC is a significant factor that should not be ignored in studies of vascular energetics.
\end{abstract}

\section{Keywords}

Myogenic Active Stress, Biomechanics, Fractal Integral, Oxygen Consumption, Allometric Law

\section{Introduction}

The energetics of the vascular system has been explored, mainly with respect to the optimum models of its

"Corresponding author.

How to cite this paper: Kamiya, A., Shibata, M. and Yamamoto, K. (2014) Assessment of Myogenic Power Expenditure Due to Arterial Wall Smooth Muscle Contraction Based upon the Fractal Nature of Vascular Trees. Applied Mathematics, 5, 1750-1762. http://dx.doi.org/10.4236/am.2014.512168 
branching system, e.g., the minimum work model by Murray [1] [2] or the minimum volume model by Kamiya and Togawa [3] and Kamiya et al. [4]. In most analyses of these models, the cost function to be minimized has been defined as the sum of the mechanical power loss due to viscous resistance against blood flow through narrow vessels and chemical energy demand to sustain massive volume of blood fresh and active in large vessels. However, one problem with these models is that the mechanical energy expenditure due to vascular smooth muscle contraction (VSMC), which consistently regulates the vascular tone and radius, has not been included in the cost function; this is in contrast with cardiac ventricular energetics [5] in which myocardial contraction is treated as an essential element in exerting mechanical power for pumping blood. VSMC has been neglected because, as demonstrated by Johnson [6], the oxygen consumption rate of VSMC per unit of mechanical power is astonishingly low, in comparison with those of cardiac and skeletal muscle contractions (interlocking mechanism). However, the significant role of VSMC in the vascular energetics should not be ruled out, until we accurately determine the magnitude of its mechanical power expenditure and assess the amount of oxygen $\left(\mathrm{O}_{2}\right)$ it consumes.

As a matter of fact, in vivo measurements of $\mathrm{O}_{2}$ tension $\left(\mathrm{P}_{\mathrm{O}_{2}}\right)$ in peripheral arterial blood in rat cremaster muscle by Shibata et al. [7] have revealed that the level of $P_{\mathrm{O}_{2}}$ in arterial blood ordinarily declines toward terminals and that the descending rate significantly diminishes when the terminal arterial VSMC is eliminated by topical application of vasodilator. These findings suggest that one of the factors inducing $P_{\mathrm{O}_{2}}$ reduction in arterial blood toward the periphery is the active consumption of $\mathrm{O}_{2}$ within the vascular wall due to VSMC [7].

To evaluate the total mechanical power due to VSMC in the entire vascular system, we need to utilize the fractal nature of the vascular branching structure introduced by Mandelbrot [8]. In our preceding study [9], we confirmed that various morphological and functional properties of the vascular system can be quantified by fractal-based integrals and their derivatives (see Appendix). Accordingly, the most urgent task to be done in this study is to construct a theoretical model for evaluating the mechanical power expended by VSMC per unit vascular wall mass and to assess its total amount in relevant vessel region using fractal integration. The outcomes of the analyses will be compared with conventionally physiological findings to verify the validity of the assessment and to substantiate the significance of this type of approaches in system physiology.

\section{Methods}

1) Theoretical assessment of mechanical power elicited by VSMC.

Figure 1 illustrates the circumferential stress-strain $(\sigma-\varepsilon)$ relationship in a vascular wall under the condition that the internal hydrostatic pressure (blood pressure, $P(r)$ ) remains constant. It is known that in such a $\sigma-\varepsilon$ diagram, the area enclosed by the trajectory of one cycle represents the amount of energy exerted in the cycle per unit mass.

The average circumferential stress $(\sigma)$ in the wall can be expressed, according to Laplace's law, as

$$
\sigma=\frac{r P(r)}{h}
$$

where $r$ is the internal radius of a cylindrical vessel and $h$ is its wall thickness. The strain $(\varepsilon)$ on the vessel wall is ordinarily expressed with the unstressed radius $r_{0}$ as,

$$
\varepsilon=\frac{r-r_{0}}{r_{0}}, \text { or } r=r_{0}(\varepsilon+1)
$$

In the present analyses, the standard state for any vessel segment is set at its maximally vasodilated state, which is the point indicated by $s$ in Figure 1. The suffix $s$ attached to parameters in the text designates those at the standard state. The radius $\left(r_{s}\right)$ in this standard state is given in terms of the corresponding strain $\left(\varepsilon_{s}\right)$ by,

$$
r_{s}=r_{0}\left(\varepsilon_{s}+1\right) \text {, and then, } r=r_{s} \frac{\varepsilon+1}{\varepsilon_{s}+1}
$$

We presume that the physiological values of $\varepsilon_{s}$ and $r_{s}$ are determined a priori for any vessel branch.

For the relation between the wall thickness $\left(h_{s}\right)$ and radius $\left(r_{s}\right)$ at the standard state, Suwa and Takahashi [10] have demonstrated that for a wide range of vessel size, $h_{s}$ can be expressed as a power function of $r_{s}$ with exponent $\theta \approx 0.71$. In addition, it was suggested that the ratio $\left(h_{s} / r_{s}\right)$ near the origin is approximately 


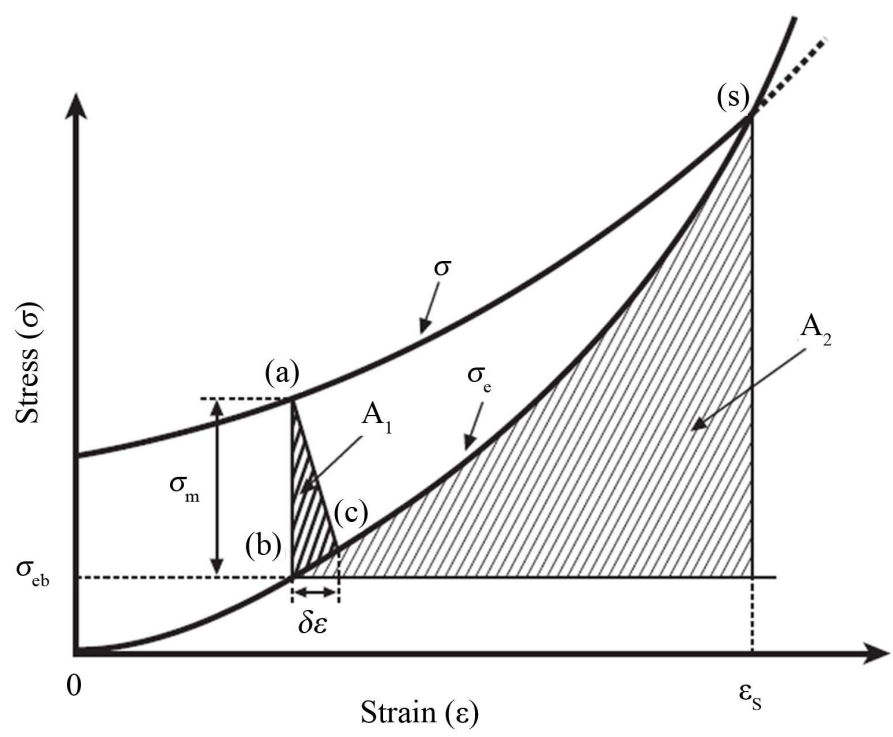

Figure 1. Stress-strain $(\sigma-\varepsilon)$ diagram for a vessel segment under the quasi-static constricting process due to vascular smooth muscle contraction (VSMC). Total stress is $\sigma=\sigma_{m}+\sigma_{e}$ where $\sigma_{m}$ is the active myogenic stress whereas $\sigma_{e}$ is the passive elastic one.

0.2 [11]. Accordingly, we have,

$$
h_{s}=0.2 r_{o s}\left(\frac{r_{s}}{r_{o s}}\right)^{\theta}=h_{0} r_{s}^{\theta}, \quad\left(h_{0}=0.2 r_{o s}^{1-\theta}\right)
$$

According to Schmidt-Nielsen [12], a number of morphological and functional properties in mammals can be expressed as the power functions of their body weights (the allometric law). The radius of the aorta $\left(r_{o s}\right)$ is also allometrically related to body weight ( $w_{b}$ in $\mathrm{kg}$ ) as $r_{o s}=0.26 w_{b}^{0.33}$. Then, the circumferential stress at the standard state $\left(\sigma_{s}\right)$ can be written as;

$$
\sigma_{s}=\frac{r_{s} P\left(r_{s}\right)}{h_{s}}=\frac{r_{s}^{1-\theta} P\left(r_{s}\right)}{h_{0}}
$$

In Figure 1, we consider a quasi-static constricting process of a vessel due to VSMC under the condition of constant internal hydrostatic pressure. In this process, which starts from the maximally dilated (standard) state, substantial changes in the entire stress $(\sigma)$ take place, because of changes in its two components, i.e., the active myogenic stress $\left(\sigma_{m}\right)$ and the passive elastic one $\left(\sigma_{e}\right)\left[\sigma=\sigma_{m}+\sigma_{e}\right]$. As VSMC is gradually enhanced, the augmented active stress $\left(\sigma_{m}\right)$ diminishes the passive stress $\left(\sigma_{e}\right)$, which accompanies continuous reductions in the total stress $(\sigma)$. Based on the assumption of constant hydrostatic pressure $\left[P(r)=P\left(r_{\mathrm{s}}\right)\right]$ and due to mass balance $\left[r h=r_{s} h_{s}\right]$, the entire stress $\sigma$ in Equation (1) can be rewritten, in terms of the standard state stress $\left(\sigma_{s}\right)$ and $\operatorname{strain}\left(\varepsilon_{s}\right)$,

$$
\sigma=\sigma_{s}\left(\frac{r}{r_{s}}\right)^{2}=\sigma_{s}\left(\frac{\varepsilon+1}{\varepsilon_{s}+1}\right)^{2}
$$

The curvature of the elastic stress $\left(\sigma_{e}\right)$ of arteries against strain $(\varepsilon)$ is also known to be well simulated by a power function of $\varepsilon$, with the exponent $(\gamma)$ in the range of $2.5-2.6$ and the maximum strain $\left(\varepsilon_{s} \approx 0.7\right)$ [13] (see Table 1). Then, $\sigma_{e}$ can be written as,

$$
\sigma_{e}=\sigma_{s}\left(\frac{\varepsilon}{\varepsilon_{s}}\right)^{\gamma} \cdot\left(0 \leq \varepsilon \leq \varepsilon_{s} \approx 0.7\right)
$$


Table 1. Values of constant parameters used in present simulation studies.

\begin{tabular}{|c|c|c|c|}
\hline Parameter & Symbol & Data & References \\
\hline The strain of the vascular wall at maximally dilated (standard) state & $\left(\varepsilon_{s}\right)$ & 0.7 & [13] \\
\hline The exponent of the radius-wall thickness relation in Equation (3) & $(\theta)$ & 0.712 & [10] \\
\hline The exponent of the elastic stress to the relative strain $\left(\varepsilon / \varepsilon_{s}\right)$ in Equation (6) & $(\gamma)$ & 2.57 & [13] \\
\hline Specific gravity of animal bod in Equation (10) & $(\rho)$ & $1.03 \mathrm{~g} / \mathrm{cm}^{3}$ & [11] \\
\hline Blood viscosity at large arteries in Equation (17) & $\left(\mu_{\infty}\right)$ & $4 \times 10^{-2} \mathrm{dyn} \cdot \mathrm{s} / \mathrm{cm}^{2}$ & {$[11]$} \\
\hline Haynes' parameter in A-14 & $(\delta)$ & $4.83 \times 10^{-4} \mathrm{~cm}$ & [9] \\
\hline Fractal dimension of systemic arterial trees in mammals in Equation (11) & $(D)$ & 1.75 & [9] \\
\hline The exponent of branch radius-length relation in A-7 & $(\alpha)$ & 1.13 & [10] \\
\hline
\end{tabular}

Using Equations (5) and (6), the active myogenic stress $\sigma_{m}$ becomes,

$$
\sigma_{m}=\sigma-\sigma_{e}=\sigma_{s}\left[\left(\frac{\varepsilon+1}{\varepsilon_{s}+1}\right)^{2}-\left(\frac{\varepsilon}{\varepsilon_{s}}\right)^{\gamma}\right]
$$

In a steady state of the wall, the constricting force due to the circumferential stress $(\sigma)$ is equilibrated with the distending force caused by the internal hydrostatic pressure. The mechanical power elicited by VSMC under such an equilibrated, stand still condition can be evaluated by considering a virtual brief cessation and recovery of the contraction and by calculating the mechanical work done by the internal pressure during the virtual cycle, as indicated by the triangle $(a \rightarrow b \rightarrow c \rightarrow a)$ in Figure 1 . The cessation of VSMC at the point $a$ reduces the stress by $\sigma_{m}$ and causes a quick shift of the operating point down to $b(a \rightarrow b)$. At that moment, the stress in vascular wall becomes off-balanced and the vessel begins to extend by $\delta r=r_{0} \delta \varepsilon$ during a brief time, $\delta t$ $(b \rightarrow c)$. Recovery of the contraction then follows, instantaneously shifting up the operating point back to $a$ $(c \rightarrow a)$. The area $\left(A_{1}\right)$ enclosed by these trajectories, $(a \rightarrow b),(b \rightarrow c)$ and $(c \rightarrow a)$ is $A_{1}=\sigma_{m} \delta \varepsilon / 2$. This represents the mechanical work done by the internal distending pressure during time $\delta t$ per unit wall mass. Obviously, the mechanical power exerted by VSMC per unit mass of wall tissue $\left(\dot{w}_{m}\right)$ is equal to the above work normalized over a unit time,

$$
\dot{w}_{m}(r)=\frac{A_{1}}{\delta t}=\frac{1}{2} \sigma_{m} \frac{\delta \varepsilon}{\delta t}
$$

The expanding rate $(\delta \varepsilon / \delta t)$ in Equation (8) is proportional to the moving velocity of the wall $u\left(=r_{0} \delta \varepsilon / \delta t\right)$ at the point $b$, which gives the kinetic energy per unit mass $\rho u^{2} / 2$ where $\rho$ is specific gravity. If cessation of VSMC continues long enough, the operating point gradually moves up from point $b$ to $s$ along the $\sigma_{e}$ curve, by expending the initial kinetic energy bestowed at point $b$. Therefore, the amount of that energy is equal to the area $A_{2}$ under the $\sigma_{e}$ curve in Figure 1:

$$
\frac{1}{2} \rho u^{2}=A_{2}=\int_{\varepsilon}^{\varepsilon_{s}} \sigma_{e} \mathrm{~d} \varepsilon-\sigma_{e b}\left(\varepsilon_{s}-\varepsilon\right)=\frac{\sigma_{s} \varepsilon_{s}}{\gamma+1}\left[1-(\gamma+1)\left(\frac{\varepsilon}{\varepsilon_{s}}\right)^{\gamma}+\gamma\left(\frac{\varepsilon}{\varepsilon_{s}}\right)^{\gamma+1}\right]
$$

Here, $\sigma_{e b}$ is a certain level of the passive stress at point $b$ with strain $\varepsilon$. Using Equations (4), (7), (8) and (9), we finally obtain the following expression for $\dot{w}_{m}(r)$ :

$$
\dot{w}_{m}(r)=k_{m} \cdot f(\varepsilon) \cdot r_{s}^{\frac{1-3 \theta}{2}} \cdot P\left(r_{s}\right)^{\frac{3}{2}}=k_{m} \cdot g(\varepsilon) \cdot r^{\frac{1-3 \theta}{2}} \cdot P(r)^{\frac{3}{2}}
$$

where,

$$
k_{m}=\left(\frac{\varepsilon_{s}}{2 \rho h_{0}^{3}(\gamma+1)}\right)^{\frac{1}{2}} \cdot\left(\varepsilon_{s}+1\right),
$$




$$
\begin{gathered}
f(\varepsilon)=\left[\left(\frac{\varepsilon+1}{\varepsilon_{s}+1}\right)^{2}-\left(\frac{\varepsilon}{\varepsilon_{s}}\right)^{\gamma}\right] \cdot\left[1-(\gamma+1)\left(\frac{\varepsilon}{\varepsilon_{s}}\right)^{\gamma}+\gamma\left(\frac{\varepsilon}{\varepsilon_{s}}\right)^{\gamma+1}\right]^{\frac{1}{2}}, \\
g(\varepsilon)=f(\varepsilon) \cdot\left(\frac{\varepsilon+1}{\varepsilon_{s}+1}\right)^{\frac{3 \theta-1}{2}}
\end{gathered}
$$

With respect to the blood pressure profile $P(r)$ in Equation (10), we can utilize A-17 in Appendix, which was derived in our preceding study [9].

Equation (10) gives the magnitude of mechanical power generated by VSMC at a branch of radius $(r)$ per unit tissue mass. This mechanical power is exerted to sustain the static equilibrium against internal hydrostatic pressure $P(r)$ at a certain strain $\varepsilon$. The formulation in Equation (10) or its modification may be feasibly applied to quantitative analyses of various biomechanical phenomena in the vascular system including the myogenic activity. In such analyses, it must be instructive, if we know the aggregated intensity of the power for all branches within a relevant range of radii or its tendency to alter from the origin to terminals. Such simulations are possible by employing the fractal nature of the vascular branching system proposed in our preceding study [9], as described below.

2) Fractal integration of the mechanical power expenditure by VSMC.

Based upon the fractal nature of the vascular tree [8], we formulate the aggregated magnitude of the mechanical power generated by VSMC for any given range of vessel radii, under several assumptions. As shown in the Appendix, our mathematical model for fractal trees [9] introduces a variable called the "aggregated branch length $l_{a}(r) \mathrm{d} r$ ", which is defined as the sum of the lengths of vessel branches in a group sorted for radius in the range $r \pm \mathrm{d} r / 2$ where $\mathrm{d} r$ is a minute change of radius. This length is expressed in the following power function of $r$ with fractal dimension $D$,

$$
\begin{aligned}
& l_{a}(r) \mathrm{d} r=k_{v} r^{-D-1} \mathrm{~d} r, \\
& k_{v}=V_{o} \frac{2-D}{\pi\left(r_{o}^{2-D}-r_{t}^{2-D}\right)}
\end{aligned}
$$

Here, $V_{o}$ is the total volume of blood in the tree, while $r_{o}$ and $r_{t}$ are the radii at the origin and terminals, respectively. These parameters are well documented in human physiology [11] and widely generalized to other mammals using power laws in terms of body weight $\left(w_{b}\right)$ (allometric rule) [12], as shown in Table 2. In Equation (11), the constant $k_{v}$ is a coefficient with dimension of length and is equal to $\lambda \kappa$ in A-5 in the Appendix. The value of the fractal dimension $D$ for the systemic arterial tree has also been estimated to be $D \approx 1.75$ in our preceding study [9].

From Equations (10) and (11), the magnitude of mechanical power elicited by VSMC in branches having radii within a minute range, $r \pm \mathrm{d} r / 2$ is given by $\dot{w}_{m}(r) \cdot 2 \pi r h l_{a}(r) \mathrm{d} r$. Then, the integrated amount of power $\dot{W}_{m}\left(r_{1}, r_{2}\right)$, generated by VSMC in the vessels within a given range of radii, $r_{1} \leq r \leq r_{2}$, is expressed as follows:

$$
\dot{W}_{m}\left(r_{1}, r_{2}\right)=\int_{r_{1}}^{r_{2}} \dot{w}_{m}(r) \cdot 2 \pi r h l_{a}(r) \mathrm{d} r=2 \pi h_{0} k_{m} k_{v} \phi(\varepsilon) \int_{r_{1}}^{r_{2}} r^{\frac{1-\theta}{2}-D} P(r)^{\frac{3}{2}} \mathrm{~d} r,
$$

provided the value of $\varepsilon$ remains constant within the range.

The integral in Equation (12) may not be solved analytically but when appropriate data for the involved parameters are available, a numerical solution may be found. In such numerical integrations, we need to employ a logarithmic transformation of the variable $r$ as $\left(z=\ln \left(r / r_{t}\right)\right)$ (see Appendix).

\section{Results}

Figure 2(a) shows distributions of myogenic power $\dot{w}_{m}(r)$ generated by VSMC per unit wall mass along the vascular radius $r$. They were calculated from Equation (10) for four different levels of the strain $\varepsilon$ using the parameters in Table 1 and human data in Table 2 for $w_{b}=65 \mathrm{~kg}$. For all $\varepsilon$ levels, $\dot{w}_{m}(r)$ values decreases monotonically with increasing radii. The curves of $\dot{w}_{m}(r)$ shift lower levels as $\varepsilon$ increased.

Figure 2(b) contains the results of the fractal integrals for $\dot{w}_{m}(r)$ from Equation (11) using the same human 


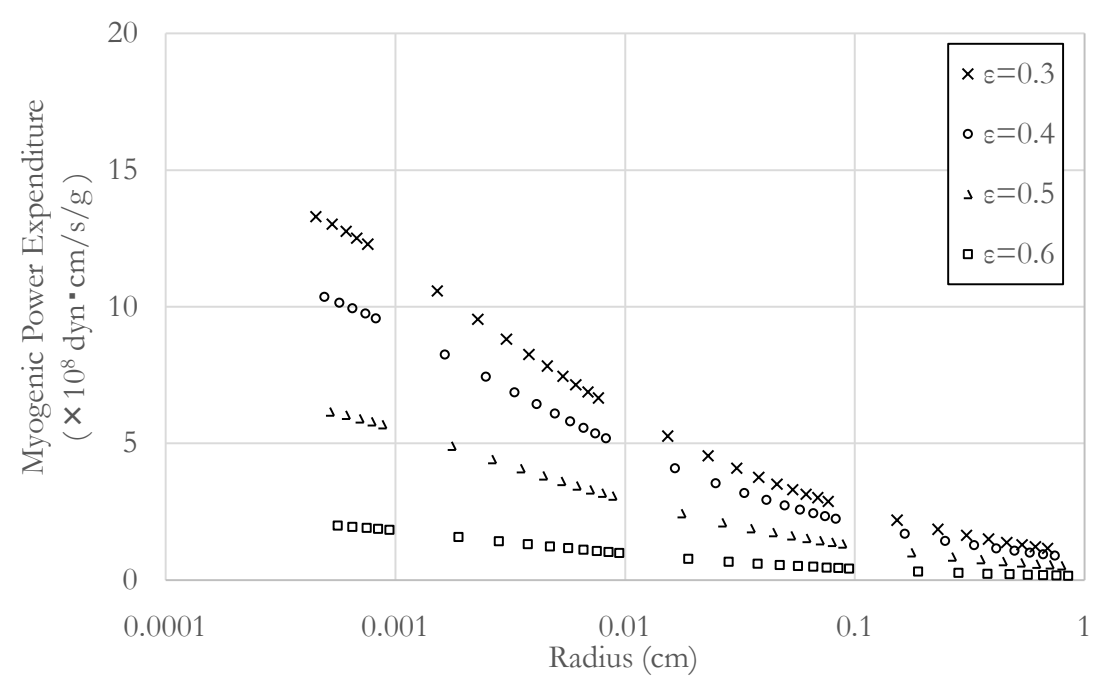

(a)

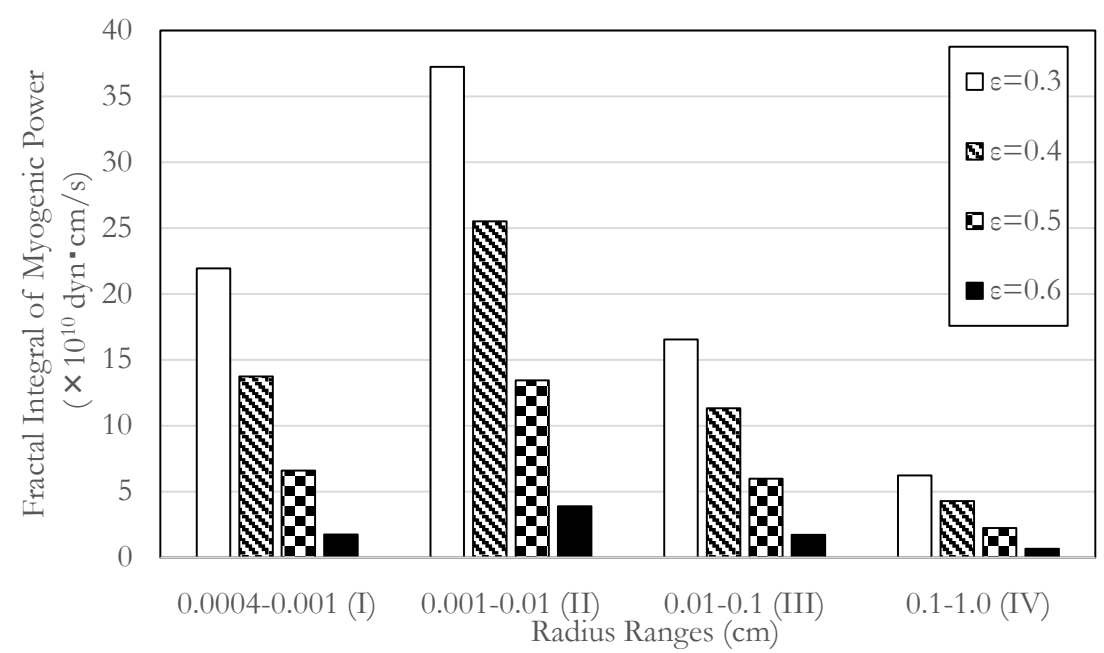

(b)

Figure 2. (a) Distributions calculated from Equation (10) for myogenic power expenditure per unit wall tissue mass $\left(\dot{w}_{m}\right)$ as functions of vascular radius $r$ for four levels of strain $(\varepsilon)$; (b) Fractal integrals calculated from Equation (12) for myogenic power over four ranges of vessel radii (segment I, II, III and IV). Calculations based on data in Table 2 for human with body weight, 60 - $70 \mathrm{~kg}$.

Table 2. Values of body weight $\left(w_{b}\right)$ dependent parameters in human $\left(w_{b}=65 \mathrm{~kg}\right)$ and in rat $\left(w_{b}=0.18 \mathrm{~kg}\right)$ employed in the analyses [4] [12].

\begin{tabular}{cccc}
\hline Parameter & Allometric equations & Data in human & Data in rat \\
\hline Arterial radius at origin $\left(r_{\text {os }}\right)$ & {$\left[r_{o s}=0.26 w_{b}^{0.33}\right]$} & $1.03 \mathrm{~cm}$ & $0.147 \mathrm{~cm}$ \\
The coefficient in Equation $(3)\left(h_{0}\right)$ & {$\left[h_{0}=0.13 w_{b}^{0.104}\right]$} & $0.201 \mathrm{~cm}$ & $0.109 \mathrm{~cm}$ \\
The arterial blood volume under vasodilatation $\left(V_{o s}\right)$ & {$\left[V_{o s}=730\left(w_{b} / 65\right)^{1.02}\right]$} & $730 \mathrm{~cm}^{3}$ & $1.80 \mathrm{~cm}^{3}$ \\
The oxygen consumption rate of the whole body at rest $\left(\dot{V}_{\mathrm{O}_{2}}\right)$ & {$\left[\dot{V}_{\mathrm{O}_{2}}=4.28 \times 10^{-1} w_{b}^{0.793}\right]$} & $11.7 \mathrm{mlO}_{2} / \mathrm{s}$ & $0.11 \mathrm{mlO}_{2} / \mathrm{s}$ \\
\hline
\end{tabular}


data as that used for Figure 2(a). The radius ranges for the integrals $\left(r_{1} \leq r \leq r_{2}\right)$ were clustered into four sections: section I $0.0004-0.001 \mathrm{~cm}$, (II) $0.001-0.01 \mathrm{~cm}$, (III) $0.01-0.1 \mathrm{~cm}$ and (IV) $0.1-1 \mathrm{~cm}$. At each level of $\varepsilon$, values of the segmental integrals $\dot{W}_{m}\left(r_{1}, r_{2}\right)$ were larger in the section II than those in the other sections.

Results corresponding to those in Figure 2 are also calculated using the rat data in Table 2 with $w_{b} \approx 0.18 \mathrm{~kg}$ and are shown in Figure 3. The values of $\dot{w}_{m}(r)$ in Figure 3(a) for rat are larger than those in Figure 2(a) for human. However, the results for segmental integrals in Figure 3(b) demonstrate that the absolute values of $\dot{W}_{m}\left(r_{1}, r_{2}\right)$ for rat are approximately 1/100 of those in Figure 2(b) for human. The values of section II are also larger than those in the other sections, although the section IV is lacking in Figure 3(b).

Based on present and reported data, we now try to estimate the coefficient between oxygen consumption rate and myogenic power expenditure due to VSMC. Using the fluorescence quenching method, Shibata et al. [7]

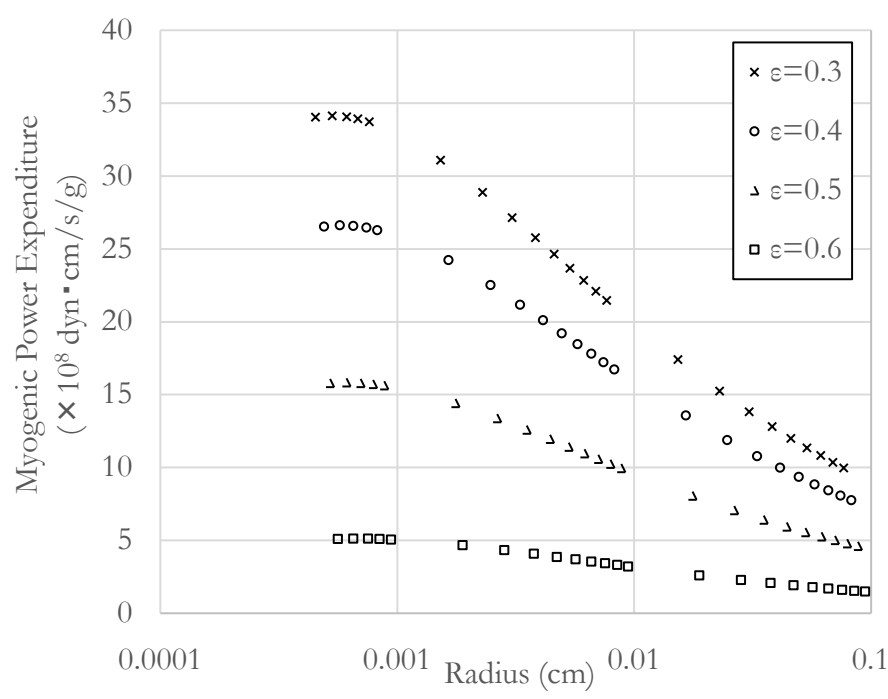

(a)

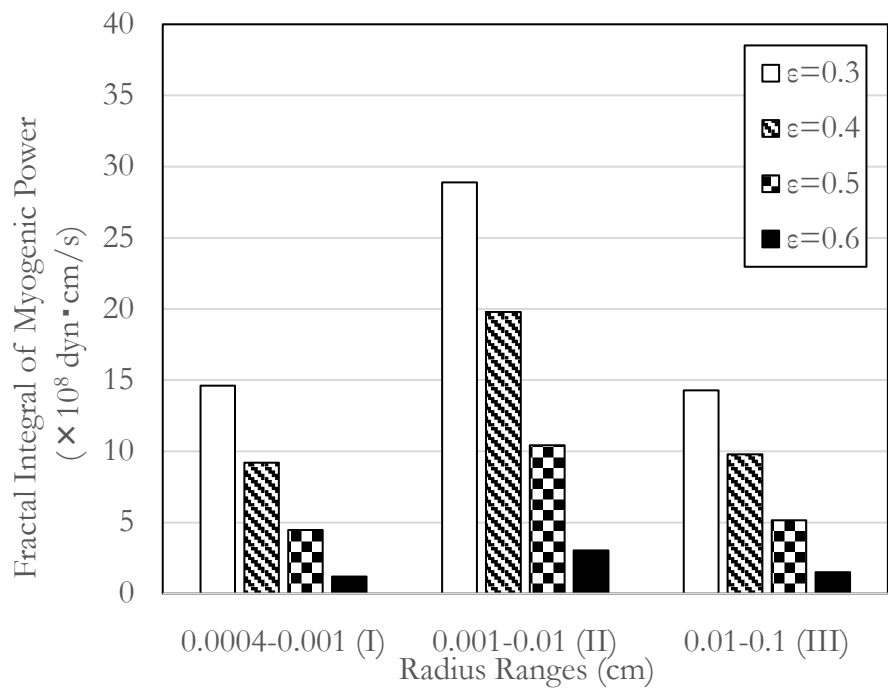

(b)

Figure 3. (a) Distributions from Equation (10) for myogenic power expenditure per unit wall tissue mass $\left(\dot{w}_{m}\right)$ as functions of vascular radius $r$ for four levels of strain $(\varepsilon)$; (b) Fractal integrals from Equation (12) for myogenic power over three ranges of vessel radii (segment I, II and III). Calculations based on data in Table 2 for rat with body weight $160-200 \mathrm{~g}$. 
measured the oxygen tension $P_{\mathrm{O}_{2}}$ in arteriolar blood and surrounding tissue in the rat cremaster muscle $\left(w_{b}=160 \sim 200 \mathrm{~g}\right)$. Then, they utilized the measured $P_{\mathrm{O}_{2}}$ to evaluate the oxygen consumption rate $\dot{q}_{\mathrm{O}_{2}}$ at arteriolar wall per tissue mass per time. Under normal conditions and during vasodilatations induced by topical application of papaverine, the averaged values of $\dot{q}_{\mathrm{O}_{2}}$ were reported to be $1.87 \times 10^{-2}$ and $0.84 \times 10^{-2}\left(\mathrm{mlO}_{2} /(\mathrm{s} \cdot \mathrm{g})\right)$, associating the averaged changes in the internal diameter from $102 \mu \mathrm{m}$ to $119 \mu \mathrm{m}$.

The differences in the two $\dot{q}_{\mathrm{O}_{2}}$ values gives the oxygen consumption rate due to VSMC $\left(\Delta \dot{q}_{\mathrm{O}_{2}}\right)$ only and the changes in diameter suggest that the strains under normal and dilated conditions were from Equation (2), $\varepsilon=0.45$ and $\varepsilon_{s}=0.7$. Then, the myogenic power expenditure $\left(\dot{w}_{m}\right)$ can be assessed from Figure 3(a) as approximately $15 \times 10^{8}(\mathrm{dyn} \cdot \mathrm{cm}) /(\mathrm{g} \cdot \mathrm{s})$. Therefore, the coefficient of oxygen consumption rate per myogenic power expenditure $\left(\Delta \dot{q}_{\mathrm{O}_{2}} / \dot{w}_{m}\right)$ is estimated as,

$$
\frac{\Delta \dot{q}_{\mathrm{O}_{2}}}{\dot{w}_{m}}=\frac{(1.87-0.84) \times 10^{-2}}{15 \times 10^{8}} \frac{\mathrm{mlO}_{2} /(\mathrm{g} \cdot \mathrm{s})}{\mathrm{dyn} \cdot \mathrm{cm} /(\mathrm{g} \cdot \mathrm{s})} \approx 6.9 \times 10^{-12} \mathrm{mlO}_{2} /(\mathrm{dyn} \cdot \mathrm{cm})
$$

\section{Discussion}

In evaluating the myogenic power expenditure due to VSMC and performing its fractal integration leading to the results in Figure 2 and Figure 3, a number of assumptions were introduced. One of the major hypotheses, which was used being in the quasi-static constriction of vascular wall in Figure 1, was that the hydrostatic pressure inside the vessel was assumed constant regardless of the extent of strain, $P(r)=P\left(r_{s}\right)$. As described in the Appendix, this condition can be achieved in large arteries by controlling the blood flow rate through each branch according to $F_{b} / F_{b s}=(\varepsilon+1) /\left(\varepsilon_{s}+1\right)$ (see (A-18)). However, in small arteries near terminals, it is not clear that the above regulation of blood flow rate is adequate, because blood viscosity becomes tube-radius dependent in small vessels (see (A-14)). Figure 4 shows calculated results for blood pressure profiles based on (A-17) at three different levels of strain, $\varepsilon=0.3,0.5$ and 0.7 , with blood flow control according to (A-18). The results in the figure reveal no discernible differences in $P(r)$ due to $\varepsilon$ levels (the maximum, $1.8 \mathrm{mmHg}$ ), suggesting that the blood pressure profile along the arterial tree is practically constant as hypothesized, so long as blood flow rate is regulated as indicated by (A-18).

The other major hypothesis used in the present calculations is that not only such univocal parameters as $\varepsilon, \varepsilon_{s}, \theta, \gamma, \mu_{\infty}, \delta, D$, and $\alpha$ but also body weight dependent parameters such as, $h_{0}, k_{m}, V_{o}, k_{v}$, and $\Delta \dot{q}_{\mathrm{O}_{2}}$ were considered to be individually fixed, uniform constants that were evenly assigned to all branches of the entire systemic arterial tree regardless of the vascular size. This presumption of uniform parameters irrespective of

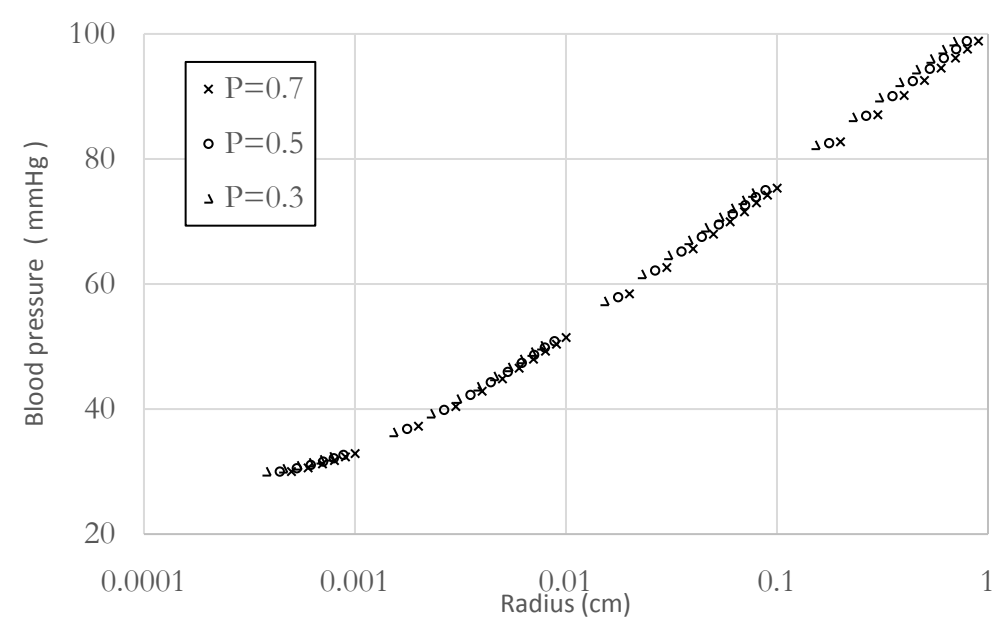

Figure 4. Profiles of blood pressure, $P(r)$, plotted against vascular radius $r$ for three different levels of strain, $\varepsilon=0.3,0.5$ and 0.7 . Pressures were calculated from (A-17) under the blood flow control based on (A-18) using human data in Table 2. The maximum pressure difference due to $\varepsilon$ values at the same standard radius $\left(r_{s}\right)$ was $1.8 \mathrm{mmHg}$. 
large and small vessels is a very bold assumption that apparently over-simplifies the physiological situations.

Nevertheless, we try to assess the total amount of oxygen consumption rate, $\Delta \dot{V}_{\mathrm{O}_{2}}$ due to VSMC in the entire systemic arterial tree in rat, by utilizing the converting coefficient, $\Delta \dot{q}_{\mathrm{O}_{2}} / \dot{w}_{m}$ obtained in the Results. The allometric equation in Table 2 and strain level, $\varepsilon=0.45$ suggest that the radius of the aorta in rat with $w_{b}=0.18 \mathrm{~kg}$ is estimated $r_{o}=0.125 \mathrm{~cm}$ whereas the terminal radius is $r_{t}=4 \times 10^{-4} \mathrm{~cm}$ regardless of body size. Therefore, the total myogenic power cost due to VSMC $W_{m}\left(r_{t}, r_{o}\right)$ is calculated from Equation (12) as $W_{m}\left(r_{t}, r_{o}\right)=29.1 \times 10^{8} \mathrm{dyn} \cdot \mathrm{cm} / \mathrm{s}$ for $\varepsilon=0.45$. Consequently, total oxygen consumption rate due to VSMC in the whole systemic arterial tree $\left[\Delta \dot{V}_{\mathrm{O}_{2}}\right]$ is calculated as,

$$
\begin{aligned}
\Delta \dot{V}_{\mathrm{O}_{2}} & =6.9 \times 10^{-12}\left[\mathrm{mlO}_{2} /(\mathrm{dyn} \cdot \mathrm{cm})\right] \times 29.1 \times 10^{8}(\mathrm{dyn} \cdot \mathrm{cm} / \mathrm{s}) \\
& =2 \times 10^{-2}\left(\mathrm{mlO}_{2} / \mathrm{s}\right)=0.02\left(\mathrm{mlO}_{2} / \mathrm{s}\right)
\end{aligned}
$$

We now compare the result in Equation (14) with the total oxygen consumption rate for the whole body in rat, $\dot{V}_{\mathrm{O}_{2}}$ in Table 2, which is given as $0.11\left(\mathrm{mlO}_{2} / \mathrm{s}\right)$ at rest [4]. These data indicate that $\Delta \dot{V}_{\mathrm{O}_{2}}$ due to VSMC only amounts to approximately $18 \%$ of the whole body oxygen consumption rate, $\dot{V}_{\mathrm{O}_{2}}$ and might exceed the cardiac oxygen consumption rate via the coronary circulation, which is estimated to be about $10 \%$ of $\dot{V}_{\mathrm{O}_{2}}$ at rest [5] [11]. Although the above ratio $\Delta \dot{V}_{\mathrm{O}_{2}} / \dot{V}_{\mathrm{O}_{2}}$ may vary according to the extent of arterial wall constrictions, it is clear that the oxygen consumption due to VSMC should be taken into account as an indispensable factor in cardiovascular energetics.

\section{Acknowledgements}

This study was supported by a Grand-in-Aid from Japan Society for Atherosclerosis Research Foundation.

\section{References}

[1] Murray, C.D. (1926) The Physiological Principle of Minimum Work I. The Vascular System and the Cost of Blood Volume. Proceedings of the National Academy of Sciences, 12, 207-214. http://dx.doi.org/10.1073/pnas.12.3.207

[2] Murray, C.D. (1927) A Relationship between Circumference and Weight in Trees and Its Bearing on Branching Angles. The Journal of General Physiology, 10, 725-729. http://dx.doi.org/10.1085/jgp.10.5.725

[3] Kamiya, A. and Togawa, T. (1972) Optimal Branching Structure of the Vascular Tree. Bulletin of Mathematical Biophysics, 34, 431-438. http://dx.doi.org/10.1007/BF02476705

[4] Kamiya, A., Wakayama, H. and Baba, K. (1993) Optimality Analysis of Vascular-Tissue System in Mammals for Oxygen Transport. Journal of Theoretical Biology, 162, 229-242. http://dx.doi.org/10.1006/jtbi.1993.1084

[5] Suga, H. (1990) Ventricular Energetics. Physiological Reviews, 70, 247-277.

[6] Johnson, P.C. (1984) The Myogenic Response. In: Handbook of Physiology, Sect. 2 (The Cardiovascular System), American Physiological Society, Bethesda, Chapter 15, 409-442.

[7] Shibata, M., Ichioka, S. and Kamiya, A. (2005) Estimating Oxygen Consumption Rates of Arteriolar Walls under Physiological Conditions in Rat Skeletal Muscle. American Journal of Physiology-Heart and Circulatory Physiology, 289, H295-H300. http://dx.doi.org/10.1152/ajpheart.00830.2004

[8] Mandelbrot, B.B. (1977) The Fractal Geometry of Nature. In: Trees and the Diameter Exponent, Freeman, New York, Chapter 17, 156-165.

[9] Kamiya, A. and Takahashi, T. (2007) Quantitative Assessments of Morphological and Functional Properties of Biological Trees Based on Their Fractal Nature. Journal of Applied Physiology, 102, 2315-2323. http://dx.doi.org/10.1152/japplphysiol.00856.2006

[10] Suwa, N. and Takahashi, T. (1971) Morphological and Morphometrical Analysis of Circulation in Hypertension and Ischemic Kidney. Urban \& Schwarzenberg, Munchen.

[11] Folkow, B. and Neil, E. (1971) Circulation. In: Vascular Length and Radius, Oxford Univ. Press, Oxford, Chapter 5, 36-56.

[12] Schmidt-Nielsen, K. (1984) Scaling: Why is Animal Size So Important? In: Heart and Circulation, Cambridge Univ. Press, Cambridge, Chapter 11, 126-142.

[13] Abe, H., Hayashi, K. and Sato, M. (Eds.) (1996) Data Book of Mechanical Properties of Living Cells, Tissues, and Organs. In: Blood Vessels, Springer-Verlag, Tokyo, Chapter 2.2, 25-114.

[14] Rosen, R. (1967) Optimality Principles in Biology. In: The Vascular System, Butterworths, London, Chapter 3, 41-60. 
[15] Haynes, R.H. (1960) Physical Basis of the Dependency of Blood Viscosity on Tube Radius. American Journal of Physiology, 198, 1193-1200.

[16] Zweifach, B.W. and Lipowsky, H.H. (1984) Pressure-Flow Relations in Blood and Lymph Microcirculation. In: Handbook of Physiology, Sect. 2 (The Cardiovascular System), American Physiological Society, Bethesda, Chapter 7, 231-307. 


\section{Appendix}

A summary of our preceding study [9] on fractal-based assessments of morphological and functional properties of the vascular system.

For a vascular system characterized by a fractal branching architecture, the probability $\Phi(r)$ that a vessel branch has a radius larger than $r$ is observed in a certain tissue region is proportional to a power function of $r$ with exponent $(-D)$. The probability can be written in terms of its probability density function $\varphi(r)$ as follows:

$$
\Phi(r) \propto r^{-D}=\int_{r}^{r_{0}} \varphi(r) \mathrm{d} r, \varphi(r)=\kappa r^{-D-1}, \kappa=\frac{D}{r_{t}^{-D}-r_{o}^{-D}}
$$

Here, $D$ represents the fractal dimension of the branching system and $r_{o}$ indicates the maximum radius at its origin. The coefficient $\kappa$ is given as above, because the minimum radii at the terminals $\left(r_{t}\right)$ are known to be uniform [12].

To intuitively perceive the fractal-based integrations of the morphological properties in the vascular system, we introduce a term "aggregated branch length" which is defined as the sum of branch lengths of vessels in a group sorted by radius around $r$ within a certain minute deviation, $\mathrm{d} r$. Evidently, the longer the aggregated branch length in a tissue region is, the more frequently the vessels in the group are observed in it. This linear relationship is simply expressed by employing the density function of the aggregated length, $l_{a}(r)$, as follows:

$$
l_{a}(r) \mathrm{d} r=\lambda \varphi(r) \mathrm{d} r
$$

where $\lambda$ is a scale factor with the dimension of length.

From (A-1) and (A-2), the aggregated branch length $\Delta L$, surface area $\Delta S$, and content volume $\Delta V$ within a certain range of radii, $r_{1} \leq r \leq r_{2}$ can be written as

$$
\begin{aligned}
& \Delta L=\int_{r_{1}}^{r_{2}} l_{a}(r) \mathrm{d} r=\frac{\lambda \kappa}{-D}\left(r_{2}^{-D}-r_{1}^{-D}\right) \\
& \Delta S=\int_{r_{1}}^{r_{2}} 2 \pi r l_{a}(r) \mathrm{d} r=\frac{2 \pi \lambda \kappa}{-D+1}\left(r_{2}^{-D+1}-r_{1}^{-D+1}\right) \\
& \Delta V=\int_{r_{1}}^{r_{2}} \pi r^{2} l_{a}(r) \mathrm{d} r=\frac{\pi \lambda \kappa}{-D+2}\left(r_{2}^{-D+2}-r_{1}^{-D+2}\right)
\end{aligned}
$$

It is now apparent that a segmental integral, $\Delta Q$ of any density function of radius $q(r)$ for a range $r_{1} \leq r \leq r_{2}$ can be expressed as, $\Delta Q=\int_{r_{1}}^{r_{2}} q(r) l_{a}(r) \mathrm{d} r$.

The expectation value of the aggregated branch length $\bar{L}_{a}(r)$ at $r$ can be obtained from (A-3) based on logarithmic sectioning of the $r$ axis as,

$$
\bar{L}_{a}(r)=\lambda \kappa r^{-D}
$$

On the other hand, Suwa and Takahashi [10] have established that the relationship between branch length $L_{b}$ and radius $(r)$ in an arterial system can be expressed as

$$
L_{b}(r)=\beta r^{\alpha}
$$

In various vascular systems, the values of the exponent $\alpha$ are clustered around 1.0. Then, the branch number $N_{b}(r)$ is given by

$$
N_{b}(r)=\frac{\bar{L}_{a}(r)}{L_{b}(r)}=\frac{\lambda \kappa}{\beta} r^{-D-\alpha}=\left(\frac{r}{r_{o}}\right)^{-D-\alpha}
$$
where $r_{o}$ is the radius at the origin, implying that $N_{b}\left(r_{o}\right)=\frac{\lambda \kappa}{\beta} r_{0}^{-D-\alpha}=1$, because the branch number at the
origin is one.

Since the radii of terminal branches $r_{t}$ are uniform in the ordinary arterial system, the number of terminals is given by $N_{b}\left(r_{t}\right)=\left(r_{t} / r_{o}\right)^{-D-\alpha}$. For the vascular system, it is well known that when a mother branch of radius 
$r_{0}$ is divided into two daughter branches of radii $r_{1}$ and $r_{2}$, they are related, with a small deviation, by a common exponent $m$,

$$
r_{0}^{m}=r_{1}^{m}+r_{2}^{m}
$$

This widely substantiated relationship is known as the "empirical power law of the vascular branching" [1] [8] [14] and the value of $m$ has been confirmed to be nearly but less than three. Note that the terminal numbers of individual trees originating from the mother and daughter branches are also related as,

$$
\left(r_{t} / r_{0}\right)^{-D-\alpha}=\left(r_{t} / r_{1}\right)^{-D-\alpha}+\left(r_{t} / r_{2}\right)^{-D-\alpha} \text { or } r_{0}^{D+\alpha}=r_{1}^{D+\alpha}+r_{2}^{D+\alpha}
$$

By comparing (A-9) and (A-10), we have,

$$
m=D+\alpha
$$

Thus, the exponent $m$ of the empirical power law in the arterial system and its fractal dimension $D$ are directly connected, with the exponent $\alpha$ of the branch length-radius relationship.

In addition, the expression $N_{b}(r)=\left(r / r_{o}\right)^{-m}$ in (A-8) renders similar formulations for cross-sectional area $\left(A_{c}\right)$, mean flow velocity $\left(U_{m}\right)$, individual branch flow $\left(F_{b}\right)$ and wall shear rate $\left(\ddot{\gamma}_{w}\right)$ as functions of $r / r_{o}$ or $r / r_{t}$ :

$$
\begin{aligned}
& A_{c}(r)=\pi r^{2} N_{b}(r)=A_{c o}\left(r / r_{o}\right)^{-m+2}=A_{c t}\left(r / r_{t}\right)^{-m+2} ; \\
& U_{m}(r)=F_{b o} / A_{c}(r)=U_{m o}\left(r / r_{o}\right)^{m-2}=U_{m t}\left(r / r_{t}\right)^{m-2} ; \\
& F_{b}(r)=F_{b o} / N_{b}(r)=F_{b o}\left(r / r_{o}\right)^{m}=F_{b t}\left(r / r_{t}\right)^{m} ; \\
& \dot{\gamma}_{w}(r)=4 F_{b}(r) /\left(\pi r^{3}\right)=\dot{\gamma}_{w o}\left(r / r_{o}\right)^{m-3}=\dot{\gamma}_{w t}\left(r / r_{t}\right)^{m-3} ;
\end{aligned}
$$

Here, $A_{c o}, U_{m o}, F_{b o}$, and $\ddot{\gamma}_{w o}$ and $A_{c t}, U_{m t}, F_{b t}$, and $\ddot{\gamma}_{w t}$ are the constant values of the individual parameters at the origin and terminals, respectively.

The profile of the blood pressure $P(r)$ is another important variable for fluid dynamics in the vascular system. The Hagen-Poiseuille law states that the pressure drop $\Delta P_{b}$ against the branch flow $F_{b}$ along a branch of radius $(r)$ and length $\left(L_{b}\right)$ is,

$$
\Delta P_{b}=\frac{8 \mu(r) F_{b}(r)}{\pi r^{4}} L_{b}(r)
$$

Here, $\mu(r)$ is the radius-dependent blood viscosity, which has been found by Haynes [15] to be well approximated by,

$$
\mu(r)=\frac{\mu_{\infty}}{(1+\delta / r)^{2}}
$$

where $\mu_{\infty}$ is the viscosity in large vessels and $\delta$ is a constant comparable with the size of red blood cells (see Table 1). Since we have $\left[\mathrm{d} L_{b}(r) / \mathrm{d} r=\beta \alpha r^{\alpha-1}\right]$ from (A-7) and $\partial P(r) / \partial L_{b}(r)=\Delta P_{b} / L_{b}(r)$ from (A13), the pressure gradient in the vascular system against branch radius, $\mathrm{d} P(r) / \mathrm{d} r$, can be written as,

$$
\frac{\mathrm{d} P(r)}{\mathrm{d} r}=\frac{\mathrm{d} L_{b}(r)}{\mathrm{d} r} \frac{\Delta P}{L_{b}(r)}= \pm k_{p} \frac{r^{m+\alpha-3}}{(r+\delta)^{2}}, \quad k_{p}=\frac{8 \alpha \beta^{2} \mu_{\infty} F_{b o}}{\pi \lambda \kappa}
$$

The symbol \pm corresponds to the arterial and venous sides, respectively. In general, the pressure profile $P(r)$ is obtained by binominal integration of (A-15). However, in our preceding studies [9], we tried curvefittings of mean flow velocity $U_{m}(r)$ in (A-12) to in vivo data measured in the peripheral vascular beds of the rat mesentery [16] and found that $D=1.75$ is the most reliable estimate of the fractal dimension $D$ for the systemic arterial tree. We also know that $\alpha=1.13$ in Table 1 is a reliable estimate by Suwa and Takahashi [10]. These two give $m(=D+\alpha)=2.88$. When these values are substituted into the exponent of $r$ in the denominator of (A-15), we have $[m+\alpha-3=1.01 \approx 1]$, indicating that (A-15) can be approximated as, 


$$
\frac{\mathrm{d} P(r)}{\mathrm{d} r} \approx \pm k_{p}\left[\frac{1}{(r+\delta)}-\delta \frac{1}{(r+\delta)^{2}}\right]
$$

By integrating the approximation (A-16), we have a simple analytical equation for blood pressure profile $P(r)$ as shown below.

$$
\begin{aligned}
& P(r) \approx P_{t} \pm k_{p}\left[\ln \left(\frac{r+\delta}{r_{t}+\delta}\right)+\delta\left(\frac{1}{r+\delta}-\frac{1}{r_{t}+\delta}\right)\right], \\
& \pm k_{p}=\frac{P_{o}-P_{t}}{\ln \left(\frac{r_{o}+\delta}{r_{t}+\delta}\right)+\delta\left(\frac{1}{r_{o}+\delta}-\frac{1}{r_{t}+\delta}\right)},
\end{aligned}
$$

where $P_{o}$ indicates the blood pressure at the origin while $P_{t}$ is the pressure at the terminals. Under normal physiological conditions, $P_{t}$ is usually regulated to be uniform. The parameter $k_{p}$ is constant with dimensions of pressure. This expression of $P(r)$ has also been confirmed to mimic in vivo data of pressure profiles measured in the peripheral vascular beds of the rat mesentery [16], both at the arterial and venous sides [9].

Another issue to consider about the blood pressure profile $P(r)$ is the assumption of constant blood pressure $P(r)=P\left(r_{s}\right)$ during the quasi-static constricting process due to VSMC, which has been introduced to deduce Equation (7) for the myogenic active stress $\sigma_{m}$. To achieve such a constant pressure situation over the entire vascular tree, it is necessary to maintain the pressure drop per unit length constant in each branch regardless of the strain $(\varepsilon)$. According to Poiseuille's law in (A-13), this condition is expressed as,

$$
\frac{\Delta P_{b}}{\Delta P_{b s}}=\frac{\mu(r)}{\mu\left(r_{s}\right)} \frac{r_{s}^{4}}{r^{4}} \frac{F_{b}}{F_{b s}}=1
$$

In relatively large vessels where $\left[\mu(r) / \mu\left(r_{s}\right) \approx 1\right]$, this condition is satisfied by adjusting the blood flow rate according to

$$
\frac{F_{b}}{F_{b s}}=\frac{r^{4}}{r_{s}^{4}}=\left(\frac{\varepsilon+1}{\varepsilon_{s}+1}\right)^{4}
$$

In small vessels in which the tube radius-dependency of viscosity is more evident $\left(r \approx \delta, \mu(r) / \mu\left(r_{s}\right)<1\right)$, changes in blood flow according to (A-18) may not guaranty a constant pressure profile. Figure 4 shows the results of the numerical calculations of $P(r)$ by (A-17) for varied strain $(\varepsilon)$, associated with the changes in blood flow $\left(F_{b}\right)$ according to (A-18), which revealed actually no discernible shifts in the profile (maximally $1.8 \mathrm{mmHg})$, even in very small arteries. 
Scientific Research Publishing (SCIRP) is one of the largest Open Access journal publishers. It is currently publishing more than 200 open access, online, peer-reviewed journals covering a wide range of academic disciplines. SCIRP serves the worldwide academic communities and contributes to the progress and application of science with its publication.

Other selected journals from SCIRP are listed as below. Submit your manuscript to us via either submit@scirp.org or Online Submission Portal.
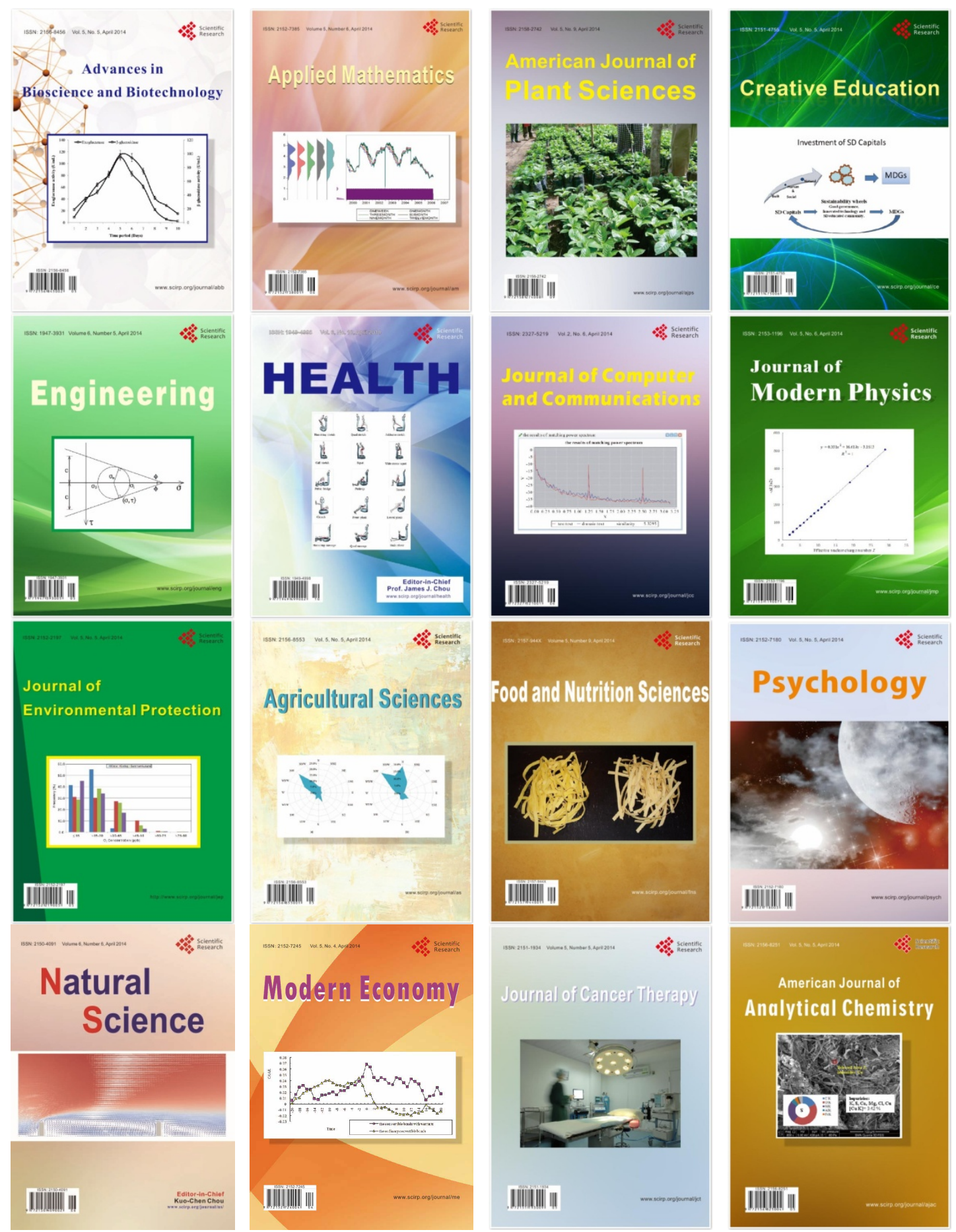